\title{
Variations
}

Variations

Revue internationale de théorie critique

$9 / 10 \mid 2007$

Les frontières de la politique

\section{L'exil du sociologue : Ernest Manheim, un migrant ordinaire}

Aldo Häsler

\section{(2) OpenEdition}

Journals

Édition électronique

URL : http://journals.openedition.org/variations/474

DOI : 10.4000/variations.474

ISSN : 1968-3960

Éditeur

Les amis de Variations

Édition imprimée

Date de publication : 1 juin 2007

Pagination : 90-105

Référence électronique

Aldo Häsler, «L'exil du sociologue : Ernest Manheim, un migrant ordinaire », Variations [En ligne], 9/10 | 2007, mis en ligne le 01 décembre 2012, consulté le 02 mai 2019. URL : http://

journals.openedition.org/variations/474 ; DOI : 10.4000/variations.474

Ce document a été généré automatiquement le 2 mai 2019.

Les ami•e•s de Variations 


\title{
L'exil du sociologue : Ernest Manheim, un migrant ordinaire
}

\author{
Aldo Häsler
}

Cousin de Karl Mannheim, Ernest (Ernö, Ernst) Manheim, sociologue, anthropologue et compositeur d'origine hongroise, fait partie de ces migrants que l'histoire a poussés de pays en pays, de recherche en recherche, de vie en vie. L'exil n'est pas toujours malheureux, comme en témoigne le parcours singulier de Manheim qui de Budapest à Vienne, de Vienne à Kiel, de Kiel à Leipzig, de Leipzig à Londres, de Londres à New York, de New York à Chicago puis finalement de Chicago à Kansas City, a adapté ses intérêts de recherche à chaque nouvelle étape de son périple, accomplissant une œuvre sociologique considérable, sans toutefois trouver dans l'ancienne Europe l'écho qui lui était dû. En marge de cette tentative de reconnaissance, je me pencherai sur la figure de la distance objectivante de l'exil et sur la plasticité particulière du sociologue traversant un siècle étrangement troublé. J'essaierai aussi de montrer que la fameuse thèse de l'« américanisation de la sociologie » qui aurait surtout frappé la France et l'Allemagne après 1945, doit être relativisée par le simple fait que la sociologie américaine a d'abord été « germanisée » (à défaut d'être gallicisée...) - et Manheim en est un exemple éloquent.

Le sociologue est un éternel migrant. Même s'il le doit parfois aux hasards de l'histoire, ou s'il se l'inflige à lui-même en guise (et en gage) de probité intellectuelle, son itinéraire est ponctué de voyages atypiques, d'arrachements inopinés, de ré-enracinements et de départs hâtifs. Comme s'il lui fallait vivre dans une éternelle zone de transit, qu'il lui fallait quitter ses parents et ses clients, ses habitudes et habitus, ses manies et ses amis, pour exercer et enrichir son regard, pour s'astreindre à une objectivité dont l'art est si difficile dans sa discipline, il est étranger à cet intellectuel en chambre ou en laboratoire, à ce savant en siège qui s'exerce à la patience et à l'accumulation lente. Certes, il est des philosophes qui voyagent, des historiens qui doivent s'exiler et même des géographes qui prennent l'avion - mais le voyage du sociologue est d'une autre nature. Que l'exil soit forcé ou non, il y a en lui une éternelle intranquillité, une exaspération à prolonger l'état de siège pour s'établir, se faire repérer, se faire assigner dans des stratégies, doublé d'une 
curiosité jamais assouvie qui lui intiment de sortir de la chambre et lui interdisent de prendre racine. Tout comme il y a des sociologues qui s'ignorent, il y a des sociologues qui ne l'ont jamais été - et c'est très souvent la sédentarité qui en est le critère. Je ne voudrais pas dire par là que le sédentaire est nécessairement mauvais sociologue; trop nombreux sont les cas - que l'on pense à Anthony Giddens, à Jürgen Habermas, à Niklas Luhmann ou à Alain Touraine - qui ont fait une œuvre souvent belle dans un vase clos. Mais qu'il s'agisse d'un exil intérieur, comme celui de Pierre Bourdieu ou d'Erving Goffman, ou d'une vie pourchassée par l'histoire, les cas sont trop nombreux pour ne pas s'apercevoir qu'il y a dans la nature du métier de sociologue une sorte de pulsion de mise à l'écart, d'observation oblique, qu'il y a la nécessité d'une diffraction qui n'est pas momentanée comme chez l'anthropologue, mais chronique et solidaire de son activité.

On a souvent considéré la période d'après-guerre comme une période d'américanisation de la sociologie allemande. Pour appuyer cette thèse, on a soutenu que le sol de cette sociologie était pratiquement vierge, qu'un véritable plan Marshall avait été développé pour ré-insuffler de la vie dans cette discipline particulièrement dévastée par le nazisme. On voudrait montrer ici, que même si ce fut le cas, cette thèse doit être relativisée. Car il faut comprendre l'interprétation de cette période comme une réponse à ce qui s'est produit en 1933, au moment où la sociologie allemande s'est autosynchronisée (selbstgleichgeschaltet) au régime nazi ${ }^{1}$. Bel euphémisme que ce terme, alors que la sociologie allemande en 1933 - ou ce qui en restait - s'est tout simplement livrée sans discussion aux forces totalitaires. Et il faut prendre en compte le fait que la majorité des chaires et des instituts de sociologie subsistent après 1945, qu'ils aient été dénazifiés ou non. D'autre part, si l'émigration des intellectuels allemands apporte à la sociologie américaine son lot de concepts, de théories et de méthodes, elle permet aussi aux émigrés de prendre connaissance du Nouveau Monde et d'y éprouver leurs outils dans des conditions toujours difficiles, mais finalement assez productives pour leurs travaux. Le sol n'est donc pas vierge, et la sociologie américaine importée n'est pas aussi américaine qu'on ne le pense. C'est en fait - en partie - une réimportation de contenus allemands apportés par les émigrants de 1933-1939.

Le cas d'Ernest Manheim n'est pas celui d'un exil malheureux². Se faire exiler est une détestation; transformer l'exil en expérience est un art auquel le sociologue ne s'est que trop souvent consacré - pour le bonheur de sa discipline. Né à Budapest en 1900, d'origine modeste, d'un père tailleur juif, Ernö Manheim est d'abord un esprit brillant avec un départ dans la vie particulièrement mouvementé. Combinant études de chimie, de physique et de mathématiques avec des études littéraires et de philosophie, embrigadé dès 17 ans dans l'armée de François-Joseph, combattant sur le front italien, fait prisonnier, puis s'évadant, puis volontaire en 1919 dans l'armée de la République des Conseils hongroise, fait prisonnier et s'évadant à nouveau, il doit émigrer en Autriche en 1920, où il finira ses études de chimie, non sans s'être frotté aux austro-marxistes autour du sociologue Max Adler. Invité par un ami à Kiel, il fréquentera Ferdinand Tönnies, notamment au temps de sa grande enquête sur le suicide dans le Schleswig Holstein ${ }^{3}$, autre étape de son initiation sociologique, avant d'entamer un cursus de philosophie auprès du sociologue philosophant Hans Freyer (1887-1969), avec lequel il se liera d'amitiét. Il soutient sa thèse de doctorat de philosophie en août 1928, avec Freyer et Theodor Litt ${ }^{5}$, thèse qui à pour titre Zur Logik des konkreten Begriffs, qui semble être (autant que la brièveté et la densité du manuscrit - 159 pages - permettent d'en juger) une tentative de fonder « existentiellement » la logique formelle. Dans la présentation de sa 
thèse, Manheim le formule de la manière suivante : «Pour penser et comprendre, il faut d'abord exister. Exister, c'est être déterminé par la réalité (Wirklichkeit) et se comporter face à celle-ci. En existant, on définit ou l'on a un rapport intentionnel à la réalité. La pensée n'est qu'un mode de l'existence; elle a une forme d'intentionnalité portée à la conscience et comprise en tant que telle. La différence entre penser et être doit être faite à l'intérieur de l'être : c'est l'être qui est surplombant. ${ }^{6}$ ». On voit donc qu'il s'agit d'une manière de se saisir de la philosophie existentielle heideggérienne pour en formuler les prolégomènes d'une méthodologie logique. Autant que l'on puisse le reconnaître, ce travail restera sans suite. Ou plutôt, Manheim - un peu à l'image de son mentor, Hans Freyer - va donner une tournure plus réelle, plus matérielle à cette thèse, et plutôt que de parler d'être et d'existant, il va se tourner vers les conditions objectives, socioculturelles des formes de la pensée. L'influence de son cousin, Karl Mannheim, qui pour sa part mène une carrière brillante, n'est pas à négliger. Car les deux ont opéré - chacun dans des contextes différents - un "tournant sociologique» de la philosophie, les menant d'un questionnement transcendantal ou existential vers une sociologie de la connaissance et un souci accru de l'empirie - tout particulièrement dans le cas de Manheim.

5 Le seul ouvrage que Manheim laissera aux sociologues est son étude sur l'opinion publique, réédité en Allemagne en $1979^{7}$. Ouvrage précurseur s'il en est, et qui, par la période envisagée - le XVIIIe siècle - s'inscrit parfaitement dans ce qu'on nomme à présent la "sociologie historique de Weimar" ». On pense bien évidemment aux travaux menés à la même époque par Bernard Groethuysen sur la formation de l'esprit « bourgeois » en France. Et l'on pense aux travaux ultérieurs de Jürgen Habermas qui, lui aussi, va découvrir ce que l'on peut considérer comme étant l'incarnation conceptuelle majeure de la modernité, la création d'un espace public. On verra qu'il y a une sorte de modernité régressive en Manheim qui, tout en abandonnant de plus en plus l'horizon historique, va devenir de plus en plus "présentiste » et empirique - est-ce là l'effet d'américanisme? Mais, en l'occurrence, cette brève étude, première en son genre, met en évidence avec une acuité exceptionnelle toute l'ambivalence que recèle cet «espace »: espace de la libre circulation de la parole, d'une part, mais aussi espace manipulatoire, construction de réalité sociale qui, quelques mois après la publication de l'ouvrage, mènera tout droit à la théorie de la propagande ${ }^{9}$. Nous allons y revenir plus longuement.

6 L'année 1933 marque, comme pour tant de ses collègues, une rupture radicale dans la vie de Manheim. Bien qu'ayant soutenu son Habilitation, enseignant à l'Université de Leipzig et tout naturellement destiné à passer après quelques années comme Privatdozent au rang d'Ordinarius, Freyer l'avertit des menaces qui pèsent sur lui et lui conseille de partir. C'est à la London School of Economics and Political Sciences qu'il va élire domicile entre 1933 et 1937, rédigeant au passage une nouvelle thèse, Security, Authority and Society. An Ethnological Introduction into Sociology (1935), sous la direction de Morris Ginsberg ${ }^{10}$, de Bronislaw Malinowski et de son cousin Karl, et survivant en étant l'assistant de ce dernier. Ce travail révèle l'extraordinaire pouvoir d'adaptation et d'intégration de Manheim. Bien que sa thèse n'ait pas été publiée, Manheim en a formulé l'argument principal dans une communication de 1942, «Risk and Social Security: Specified and Non-Specified Risks », donnée à Kansas City ${ }^{11}$.

7 Dans une veine bien fonctionnaliste, Manheim inverse l'argument de Thorstein Veblen sur la «leisure class». Recourant à un large matériau ethnographique, il entend montrer que dans de nombreuses sociétés traditionnelles l'existence d'une telle classe «de loisirs » ne répond pas ou pas exclusivement à des impératifs de prestige, en mettant en 
place des vecteurs de stratification, mais constituent des ressources sociales mobilisables par temps de crise. Alors que ces sociétés sont prêtes à faire face à des risques ordinaires ( "specified risks») par des routines ordinaires, quand surgissent des risques sortant de ce cadre, ce sont précisément ces classes qui sont appelées dans des fonctions économiques et militaires ; classes auxiliaires, en quelque sorte, dont Manheim se demande à juste titre si elles assurent encore cette fonction dans le cadre de la modernité. Manheim n'est pas le seul à parler de cette problématique du risque, tant s'en faut. Ainsi, l'économiste américain Frank H. Knight avait déjà, une dizaine d'années auparavant, entrepris de justifier la prise de bénéfices dans l'activité capitaliste moderne par la permanence du risque et de l'incertitude ${ }^{12}$. La veine adoptée par Manheim ne va pas dans ce sens; elle s'inscrit dans une critique de la "stérilité » (au sens saint-simonien du terme) des « classes stériles » modernes. Alors que dans les sociétés traditionnelles, l'existence d'une accumulation somptuaire fonctionnait comme une « réserve sociale » activable par temps de risque, l'accaparation propre à l'« individualisme possessif » (C.B. MacPherson) ne joue plus qu'un rôle purement « véblénien »; c'est le somptuaire pur.

Parallèlement, il réalise une étude commandée par Friedrich Pollock, le bras droit de Max Horkheimer, sur la famille autoritaire - précisément l'un des principaux sujets sur lesquels va se pencher Theodor W. Adorno durant son exil américain ${ }^{13}$. Cette Geschichte der autoritären Familie, un typoscript de 337 pages, est aujourd'hui perdue et il n'en subsiste qu'une partie, sous forme d'une contribution sous le titre «Beiträge zu einer Geschichte der autoritären Familie» dans l'ouvrage monumental édité par Max Horkheimer Studien über Autorität und Familie. Forschungsberichte aus dem Institut für Sozialforschung (Paris, Alcan, 1936, pp. 523-574) réédité en 1987 (Lüneburg, Zu Klampen). On imagine la productivité de ces années londoniennes (1933-1937). Sous la férule de son cousin et dans l'environnement d'une London School qui va peu à peu se peupler des plus illustres intellectuels du XXe siècle - que l'on songe à Karl Popper, Ludwig von Mises ou Friedrich A. Hayek, ou quelques années plus tard à Ernest Gellner -, Manheim va non seulement développer d'importants travaux sur des registres très différents, mais s'initier à l'anthropologie et à l'histoire, avant de reprendre son bâton de pèlerin...

Il faudrait un jour systématiser l'histoire du destin "américain" (ou suédois, égyptien, turc ou australien) des sociologues chassés par les Nazis ${ }^{14}$ - je ne parle ici que des sociologues, car il me semble que le cas des philosophes (pensons à Ernst Cassirer ou à Alfred Sohn-Rethel), des psychologues (pensons à Wilhelm Reich ou Alfred Adler) ou des historiens (pensons à Ernst Kantorowicz ou Raymond Klibansky), pour ne pas parler des politologues, juristes ou architectes, est à la fois plus complexe, plus massif et plus radical dans le mode de l'exil que celui de leurs collègues. Il y eut des exils définitifs et, somme toute, heureux, comme ceux de certains membres de l'École de Francfort (Herbert Marcuse, Erich Fromm ou Leo Löwenthal); il y eut des exils définitifs doublés d'oublis tout aussi définitifs comme ceux de Gottfried Salomon-Delatour ou d'Emil Lederer; il y eut des retours plus ou moins heureux comme celui de Siegfried Landshut ou d'Alexandre Rüstow ou d'autres membres de l'École de Francfort ${ }^{15}$, mais des retours ravageurs aussi; le plus scandaleux étant toujours celui d'un esprit brillant dont la carrière fut interrompue en 1933 et qui, à son retour, voit son poste occupé par le nazillon reconverti (et souvent d'une médiocrité affligeante) qui le lui avait ravi quinze ans auparavant ${ }^{16}$.

10 Ernest Manheim n'entre dans aucune de ces catégories. Il ne s'est d'ailleurs jamais considéré comme un exilé, mais a parlé de lui-même comme d'un émigrant. L'exilé est poussé hors de chez lui par une force ennemie ou par un ostracisme majeur, alors que l'émigrant choisit de partir sous d'autres cieux - go west, young man. Or il est clair que 
l'émigration de Manheim ne fut pas choisie, mais subie, et que son épopée n'est même pas due à des motifs économiques ou politiques, mais qu'on lui a fait endosser les habits du Juif errant, de celui qui n'a pas d'autre choix que de repartir sans cesse. Les contacts de Manheim avec d'autres sociologues émigrés furent multiples, notamment avec Norbert Elias (1897-1990), Hans H(einrich) Gerth (1908-1978), Rudolf Heberle (1896-1991), Eduard Heimann (1889-1967), Adolph Lowe (i.e. Adolph Löwe ; 1893-1995), Charlotte Lütkens (1896-1967), Karl Mannheim (1893-1947), Franz L(eopold) Neumann (1900-1954), Hans Speier (1905-1990), Karl August Wittfogel (1896-1988) ou Kurt Heinrich Wolff (*1912). Mis à part Franz Neumann, qui en reste un épigone, les contacts avec l'École de Francfort sont absents. C'est certainement moins dû à une frilosité de la part de Manheim qu'au fait que les membres de l'École ne cherchaient pas outre mesure le contact avec la diaspora allemande. La manière dont Horkheimer et Pollock ont cultivé une sorte de splendide isolement - munis du trésor de guerre de Felix Weil, le mécène de l'École - n'est pas l'un des chapitres les plus glorieux des Francfortois en exil. Mais cela est dû aussi à l'orientation générale de la sociologie allemande de l'époque, dans laquelle l'École de Francfort peine à s'inscrire.

11 La sociologie historique est l'une des deux grandes tendances de la sociologie allemande après la mort de Simmel et de Weber - l'autre étant la sociologie formelle dont les représentants sont Leopold von Wiese et Alfred Vierkandt. Elle s'inscrit au creux du doute révolutionnaire qui réoriente l'analyse du capitalisme. C'est là l'enjeu central de cette sociologie historique. On se méprend d'y voir, comme l'a fait René König, une simple critique morale. Le doute révolutionnaire, le soupçon quant à la possibilité du Grand Soir, n'est pas seulement dû au fait que la révolution s'est déroulée là où on ne l'attendait pas, mais à la formidable résistance qu'offre le capitalisme à toutes les crises qui l'assaillent et d'autant plus fortes que celui-ci est «avancé ». C'est là que s'enracinent deux idéesforces qui vont orienter les débats: l'ultrastabilité de ce système social, mais en même temps sa contingence, sa non-nécessité historique. On peut trouver dans ce programme de recherches la même structure logique que celle qui se développe parallèlement dans l'Anthropologie philosophique. Ce n'est évidemment pas la place de l'Homme dans le cosmos, mais celle de la modernité dans l'histoire, qui est ici le sujet de réflexion; une place aléatoire, mais en même temps d'une puissance supra historique. Tout se passe comme si le système capitaliste moderne ne pouvait plus être ébranlé, et que l'humanité se trouverait condamnée à la monotonie d'une éternelle protohistoire. Les œuvres de Werner Sombart et de Franz Oppenheimer sont là pour témoigner de cette incessante interrogation, de ce doute impérieux qui assaille les sociologues de cette époque : qu'on ne connaît ni les racines, ni le dynamisme du capitalisme et de la modernité ; doutes sur la validité de la théorie marxienne de la crise et donc doute sur son analyse sociologique du capitalisme; doute non idéologique, mais factuel sur son increvable nature. La synthèse de Marx et de Weber, que l'on connaît sous la théorie lukacsienne de la réification - qui constitue le «noyau paradigmatique » (Hauke Brunkhorst) de l'École de Francfort -, a son pendant critique dans la sociologie historique de Weimar. Les sources en sont identiques, mais l'analyse sensiblement différente. Alors que l'École de Francfort cherche désespérément à comprendre l'absence de potentiel révolutionnaire, la sociologie historique de Weimar s'interroge au contraire sur la résistance particulière qu'offre le système capitaliste moderne à toutes les crises qui le défient, voire comment ce système s'en trouve encore solidifié. 
12 L'œuvre de Manheim se place sans conteste dans le sillage de son illustre cousin. Dans la lettre de recommandation qu'il lui confie, lors de leur émigration en 1933, Karl Mannheim souligne l'originalité des recherches d'Ern(e)st en ces termes : « Le problème sur lequel il s'est penché concerne une question très largement éludée par ses collègues, à savoir le rôle des groupes sociaux dans la formation de l'opinion publique et pour l'intégration sociale $17^{17}$ ». L'objet de son travail d'habilitation concerne une question bien précise: comment se fait-il qu'en dépit d'un programme politique à peu près identique, les national-socialistes de la NSDAP remportent une victoire aussi éclatante sur le parti national allemand (DNP) dans les élections fatidiques de 1933 ? Comment expliquer que la DNP n'a finalement atteint qu'une petite couche d'électeurs, alors que la NSDAP a remporté le succès que l'on sait? Pour y répondre et conformément à la méthode de la sociologie de Weimar, Manheim va interroger la formation de l'espace public en Allemagne au XVIIIe siècle. Ce qui l'intéresse, c'est d'une part le rôle qu'y jouent certains groupes sociaux, comme les francs-maçons, les premiers publicistes, l'avantgarde artistique etc. et, d'autre part, la formulation d'une "fiction romantique » qui envahit cet espace public vers la fin du XVIIIe siècle. Le parallèle avec 1933 lui semble édifiant: car la NSDAP s'est saisie de cette même fiction pour instrumentaliser le «mouvement de jeunesse » (Jugendbewegung) d'après-guerre en profitant de son opposition à la vie bourgeoise pour lui inculquer certaines valeurs fondamentales du mouvement nazi (retour à la communauté de sang, imposition d'un leader charismatique, esprit de sacrifice, héroïsme germanique, etc.).

Manheim se place donc à la fois dans le sillage de Karl, d'une sociologie de la connaissance à base empirique, mais en lui ménageant des inflexions majeures. Pas question pour lui, l'ancien socialiste des conseils, de cautionner l'élitisme de son cousin pour qui l'intellectuel libre de toute attache (freischwebende Intelligenz) a pour fonction d'éduquer le corps social et de mener politiquement les masses. Alors que Karl adopte encore une attitude "mandarinale », largement répandue dans la sociologie de Weimar ${ }^{18}$, Ernest est très réservé sur la capacité intégrative de cette «intelligence». Ses travaux sur l'émergence du mouvement nazi lui donnent d'ailleurs raison : ce n'est pas le « one-step flow » de la voix du maître qui va mobiliser les groupements nazis en une unité massifiée, mais le relais pris par les leaders d'opinions dans les divers groupuscules. De même, il adopte par rapport à l'opinion publique une position non plus normative, visant à la former et à la transformer à la guise de l'idéologie à promouvoir, mais le regard distancié de l'observateur qui veut voir son mode de fonctionnement précis. En ce sens, Manheim est le premier à avoir cherché à coupler une sociologie de la connaissance avec une sociologie de la communication. Dans l'ensemble de ses études domine le souci de trouver la manière dont se construit la connaissance (l'opinion, les stéréotypes, les normes et les valeurs) en fonction des conditions sociales concrètes, souvent "groupales " (Didier Anzieu) de son élaboration. C'est là l'un des principaux fils conducteurs dans l'œuvre de Manheim. Depuis sa thèse de doctorat jusqu'à ses travaux sur le rapport entre schizophrénie des jeunes et contexte social, son questionnement aura toujours conservé sa structure initiale : quel rapport peut-il y avoir entre les contenus de connaissance, les définitions de réalité et la perception de soi, d'une part, et les groupements sociaux particuliers dans lesquels les individus sont pris, de l'autre? Et, à l'instar de la construction sociale de l'opinion publique, de quelle manière précise ces contenus de connaissance s'élaborent-ils au sein de ces groupes? À la différence de Mannheim, l'accent est mis ici sur une construction intersubjective et discursive de la réalité, mais 
une construction très en retrait par rapport au déterminisme social. Ainsi dans ses travaux sur les jeunes schizophrènes est-il conduit à réviser le jugement de ses collègues sociologues qui avaient tendance à imputer cette psychopathologie au déclassement social des jeunes et à l'absence des pères; ses propres recherches révèlent que ce déclassement est consécutif à la psychopathologie, mais que celle-ci doit encore être recherchée dans les cadres de la socialisation de ces jeunes. Plutôt donc que de tomber dans les travers de la psychologisation, Manheim, tout en dénonçant un sociologisme facile, reste une fois encore fidèle à son questionnement initial. La question de l'autorité, thème principal de ses recherches durant son séjour londonien, est liée à ses travaux sur la construction de l'opinion. N'oublions pas le contexte historique : voici un petit parti d'obédience ultranationaliste qui est parvenu non seulement à mettre dans l'ombre le tout-puissant parti du maréchal Hindenburg, mais qui parvient à imposer son autorité à un rythme fulgurant; imposer son autorité et imposer un "état autoritaire " (Ernst Forsthoff) sans que les contre-pouvoirs démocratiques ne puissent réagir. Et il n'est pas anodin que Manheim mène son enquête sur deux fronts, apparemment disjoints, qui lui permettent de cerner cette "obédience » de manière originale : d'une part il fait, tout comme Erich Fromm, l'hypothèse d'un apprentissage de cette soumission dans le cadre de la socialisation familiale ; et d'autre part, après avoir fréquenté les genii loci de chacune des étapes de son émigration, il eût été étonnant qu'à Londres il ne s'inspirât pas de Bronislaw Malinowski; et donc d'approcher cette soumission à l'autorité dans une perspective anthropologique. L'autorité, pour Manheim, n'est pas une catégorie abstraite, n'est pas un potentiel au sens wébérien du terme: la "chance» d'imposer sa volonté contre la volonté d'autrui. Elle est imposition de la volonté sans prendre en compte la volonté d'autrui; elle n'a nul besoin de croyance comme base de légitimation, mais impose l'obéissance aveugle. L'histoire compte pour beaucoup dans cette conception, et si Weber avait connu le phénomène nazi, il y a de fortes chances qu'il lui aurait fallu reconsidérer sa conception du pouvoir. Pour Weber, tout pouvoir doit s'établir sur une croyance, et cette croyance a toujours sa part de rationalité. Les obéissants doivent pouvoir formuler eux-mêmes les (bonnes) raisons qui les font suivre une autorité qui leur est extérieure. C'est soit la croyance en une tradition, soit la persuasion dans l'efficacité du pouvoir, soit le charisme. L'exemple de Hitler montre, en revanche, que ce dernier impose une autorité beaucoup plus inconditionnelle que les deux premières, car la transe charismatique n'a pas besoin de justification. Or, dans les sociétés traditionnelles, cette inconditionnalité, cette soumission aveugle, est réservée à des situations bien précises, à des risques «spécifiés ». Une fois l'état d'exception achevé, l'autorité rentre dans le rang et les routines reprennent leurs droits. Dans une modernité où l'autorité, conformément au bon mot de Carl Schmitt, se confond avec le droit de décréter l'état d'exception, la définition de cette exception relève de l'arbitraire. Sans le besoin de se justifier et avec la capacité de le décréter à tout moment, le pouvoir d'exception prend ainsi un aspect de permanence qui est en complète opposition avec le despotisme traditionnel. L'hypothèse qui viserait à expliquer qu'une telle soumission inconditionnelle n'est possible que par un dressage préalable est donc tout à fait cohérente. Pour être "naturelle », l'obéissance aveugle, ce blinder Gehorsam qui est devenu le stéréotype du nazi, doit être infligée tout au début de la socialisation. Voilà qui explique cet intérêt pour l'éducation autoritaire.

Lorsqu'il passe à Chicago, en 1937, Manheim n'a de cesse de s'approprier les méthodes de l'École du même nom, ce qui fait de lui un sociologue muni d'une solide formation philosophique, avec des objectifs théoriques qu'il tente d'évaluer en recourant à toute la panoplie d'outils empiriques; un «allround-man » sociologique en quelque sorte, qui 
draine sur son passage les acquis intellectuels du Mitteleuropa, de la théorie sociale allemande, de l'anthropologie sociale britannique et finalement de la sérendipité de Chicago ; et c'est muni de ce bagage qu'il ira s'établir dans le Missouri, à Kansas City, où il enseignera de 1938 à 1991, en ayant fondé et dirigé le Département de sociologie de l'Université de Kansas City (UMKC) entre 1948-1970. Une extraordinaire longévité, un record d'enseignement - 53 années passées dans le même Département universitaire - et surtout un travail de formation d'une remarquable constance: on évalue à 2500 les étudiants qui ont bénéficié de ses cours et de ses innombrables polycopiés (non publiés à ce jour). Manheim fut l'un des artisans de l'American Journal of Sociology qu'il contribua à lancer et auquel il consacra un nombre considérable de notes de lecture - témoignant par là encore de ce souci artisanal et de son retrait par rapport aux gloires académiques. Ses premiers travaux s'impliquent directement dans la réalité américaine : ainsi, il étudie le rapport entre les pathologies mentales des adolescents et la vie citadine, initie les premières " community studies ", notamment sur les modèles de proximité de voisinage (community councils) et entreprend des recherches étendues sur les préjugés et les minorités. Kansas City devient son laboratoire. Pendant la guerre, il s'implique activement dans le travail social visant les jeunes privés de parents - le père au front, la mère engagée dans l'effort de guerre. Tout en menant ses travaux empiriques, il va défendre après 1945 la tradition sociologique allemande, tout comme le feront Kurt $\mathrm{H}$. Wolff ou Hans H. Gerth, ce qui fera de Manheim l'un de ces nombreux passeurs qui, bien avant l'américanisation de la sociologie allemande ont contribué à insuffler à la sociologie américaine la rigueur méthodologique, le souci conceptuel et analytique et l'originalité interdisciplinaire qui ont fait la force de cette tradition sociologique. Par bien des aspects, la fameuse «américanisation » de la sociologie allemande n'est qu'une réimportation de produits intellectuels allemands passés au tamis d'une organisation scientifique nouvelle - comme en témoignent par exemple la trajectoire et la carrière de Paul F. Lazarsfeld. Car si l'on parle à juste titre de la tragédie qu'a constitué l'exil à partir de 1933, on oublie bien souvent les assimilations heureuses et tout l'apport que les exilés ont fourni à une sociologie américaine encore très largement artisanale.

La sociologie historique de Weimar dut son déclin à trois facteurs : d'une part au nazisme dont l'idéologie anti-capitaliste avait promis le dépassement du système honni et pour laquelle les monumentales fresques sombartiennes devenaient parfaitement inutiles; ensuite, l'américanisation de la sociologie allemande après 194, réclamant une sociologie appliquée et technocratique; en troisième lieu, on s'aperçoit qu'une grande partie des sociologues allemands issus de cette école s'enracinant sur le sol américain tourna les dos à ce type de préoccupation encyclopédique pour se consacrer à des études plus conjoncturelles. Manheim est de ceux-là. Alors que le «noyau paradigmatique » lukacsien de l'École de Francfort recelait suffisamment d'ambiguïtés et de problèmes pour sauvegarder le « programme de recherches » d'origine, la sociologie de Weimar, de par sa perspective plus historiste, menait nécessairement à une plus grande dissémination des interrogations. Transplantée hors de son cadre d'origine, elle perdait ainsi de son unité et de sa pertinence.

Les émigrants allemands ont plus contribué à l'étude et au rayonnement de la tradition sociologique allemande que tous les autres sociologues réunis. Il s'agit là d'un effet de diaspora assez typique où, l'éloignement aidant, on se réserve une portion de patrie intellectuelle dans un exil dont on ne connait jamais la durée. Tout comme Hans Gerth ou Kurt Wolff, Ernest Manheim s'est fortement impliqué dans cette réécriture de l'histoire 
de la discipline. En 1948, il publie la première étude en langue anglaise sur les travaux sociologiques de son ancien maître, Hans Freyer, dans le manuel de Harry Barnes, An Introduction to the History of Sociology, dont la portée pour la formation et la prise de conscience d'une «tradition sociologique » fut considérable. Freyer dont le passé nazi va interrompre la carrière, et qui trouvera une audience beaucoup plus grande parmi les historiens allemands - il est parmi les fondateurs, avec Werner Conze, de la nouvelle histoire sociale allemande - obtiendra ainsi un nouvel écho et un regain d'intérêt, notamment lorsque reprennent les études en sociologie industrielle, dans les années 1950, avec les travaux d'un Friedrich Jonas. Plus importants encore, puisqu'il en assure l'édition intégrale, les Collected Papers de son cousin Karl sont devenues la référence des études mannheimiennes. Tout se passe comme si Manheim, une fois son objet étudié de manière qui le satisfasse, l'abandonne ensuite pour se tourner vers d'autres chantiers. La logique existentielle, l'opinion publique, l'anthropologie des risques, la famille autoritaire - voilà autant d'objets d'une trajectoire apparemment disparate où le souci de l'objet l'emporte sur l'idée d'une œuvre à accomplir. Mais c'est dans son enseignement que Manheim a placé l'essentiel de ses forces, dans le travail quotidien avec ses étudiants.

17 Manheim ne prit pas la trajectoire "multinationale " d'un Lazarsfeld. Alors même que son souci empirique allait s'accroissant au fil des ans, Manheim ne croisa pas les chemins de son illustre confrère de Columbia. Les deux austro-hongrois se sont bien connus, pourtant. Au temps où Lazarsfeld faisait ses premières armes de psychologue social après une thèse de mathématiques - chez Charlotte Bühler, Manheim s'initiait à la philosophie. Les contacts épistolaires sont assez fréquents entre les deux ; très souvent Manheim corrige des articles de Lazarsfeld et lui envoie les siens pour avoir ses commentaires. Néanmoins, ils n'évoqueront jamais une collaboration et, d'après mes connaissances, Lazarsfeld ne citera jamais Manheim dans sa production profuse. De même, il est évident que le « one-step floc », qu'on appelle parfois « Loi de Lazarsfeld » le fait que les mass media n'ont qu'une influence indirecte sur l'opinion publique (ici, les intentions de vote) et qu'ils doivent être relayés par des leaders d'opinion -, comme l'expose Lazarsfeld dans son ouvrage People's Choice ${ }^{19}$ avait été clairement développé en 1939 par Manheim dans plusieurs publications ${ }^{20}$; il nous semble peu vraisemblable que Lazarsfeld n'en ait pas eu connaissance. Manheim ne s'arrêtera pas là ; on lui doit des études sur la dissonance cognitive bien avant les travaux de Leon Festinger, et même si Kurt Lewin est à juste titre le découvreur de la théorie du " gate-keeper »- celui qui, dans les petits groupes, garde les canaux, les entrées et sorties -, c'est encore Manheim qui va préciser cette théorie et l'appliquer à ses terrains. Il est évidemment difficile de reconstruire à travers la multitude de travaux épars l'unité d'une théorie que son auteur n'a jamais synthétisée, pire, qu'il a laissé lui-même à l'état de contributions anonymes au progrès de sa discipline. Mais il ne saurait faire de doute qu'à travers la reformulation discursive de la sociologie de la connaissance, Manheim a fait un pas important vers une théorie sociologique de la communication. La masse de travaux d'apparence hétéroclite et souvent attachés à un objet précis ne doit pas occulter la trame de fond de toute sa démarche: Manheim veut de toutes les manières possibles - par la comparaison historique et anthropologique, la critique épistémologique et le patient travail empirique - relativiser le substantialisme sociologique, la Seinsgebundenheit de la connaissance. En recourant à l'approche communicationnelle et discursive, ce qui l'intéresse, c'est l'élaboration interrelationnelle des contenus de conscience. Cette critique implicite du modèle de Shannon et Weaver repose sur ce qu'on appellera plus tard, à la suite de Luhmann, le problème de la « double contingence ». L'émetteur n'adresse pas un message 
sans prendre en considération ce qu'il pense pouvoir évoquer chez le récepteur, mais l'inverse est également vrai, et l'émetteur devra prendre en compte cette double anticipation dans un jeu de spéculations croisées qui ne connaît théoriquement pas d'issue. Or, et c'est là où le pragmatisme américain va influencer Manheim, cette issue n'est pas problématique en tant que telle, et si elle ne l'est pas, c'est que les acteurs mettent en oeuvre toute une série d'approximations réciproques qui leur permettent de trouver une issue pratiquement uno actu dans leurs communications. C'est cette élaboration sociale et intersubjective de solutions pratiques du problème de la « double contingence " que Manheim va tenter de déchiffrer dans une grande partie de ses travaux.

Manheim s'enracina à Kansas City. Alors que ses confrères allaient fréquenter les régions côtières et les universités réputées, Manheim va jeter son dévolu sur le Middlewest, sur une université de «moyenne portée » et une ville à visage humain, dans une stratégie de retrait qui n'a pourtant rien d'un renoncement. Mais il ne participe pas aux grandes joutes paradigmatiques, le systémisme ne l'effleure même pas, de même que les nouvelles tendances de l'interactionnisme. En dépit d'une formation confiée aux intellectuels les plus illustres du Mitteleuropa - qu'on songe à Tönnies, Freyer, Litt, Mannheim, Ginsberg, Malinowski, pour ne citer que les plus connus -, sa brève incursion à Chicago en aura fait un « sociologue-praticien ». On connaît d'ailleurs ses trois passions outre la sociologie : la marche à pied, l'ébénisterie et la composition musicale. On n'évoque guère les violons d'Ingres dans la confrérie des sociologues, mais ceux de Manheim sont suffisamment originaux pour être relevés : ainsi parcourt-il de larges parties des Etats-Unis à pied, confectionne-t- il lui-même l'intégralité de son mobilier. Et il reste sa passion profonde pour la musique où, dans une veine dodécaphonique et dans la continuité de ses héros que sont Webern et Berg, il compose concertos et symphonies d'une facture qui est tout sauf l'œuvre d'un dilettante.

19 La ritournelle du sociologue méconnu a été suffisamment entonnée pour ne pas s'y consacrer une fois encore. D'ailleurs Manheim s'est délibérément détourné de toute production théorique dès 1933. Son parcours - des hautes sphères spéculatives aux travaux sur les «community councils» - est lié à sa profonde déception face à la pensée allemande; et il a été conséquent jusqu'au bout: une pensée aussi grandiose qui ne saurait empêcher la pire barbarie de s'accomplir n'a selon lui aucun intérêt. Son regard d'exilé est donc celui du Zaungast, de celui qui assiste de loin aux grands débats, sans toutefois émettre de jugements, mais en faisant simplement son travail. Même au grand âge, ce regard distancié ne l'a pas quitté. Pour lui, même si l'exil a été une nécessité, il l'affronte sans le pessimisme culturel des Allemands, le faisant plus découler d'un choix personnel que d'une fatalité historique. Cette absence d'exemplarité, ce retrait de la personne devant le travail à accomplir, ce refus du geste magistral - tout cela aura peutêtre nui à la réception d'une oeuvre importante. Mais devant le choix de se poser en victime et de donner des leçons ou d'être anonymisé par le succès de son travail, Manheim a constamment choisi cette seconde posture. Le regard de l'exilé n'est pas nécessairement torturé. Nous avions dit au départ que la sociologie est par excellence une pensée nomade, et il est vrai qu'à force d'être poussé d'un siège à l'autre le regard distancié du nomade s'émousse et se charge de ressentiment. Rien de tel chez Manheim qui a choisi le «middle range » si cher à Robert $\mathrm{K}$. Merton, non pour encenser le statu quo social, mais pour y exercer sa sagacité et son ironie. À la ritournelle de la méconnaissance, Manheim nous aurait renvoyés à nos propres fantasmes en nous 
enjoignant probablement de faire une cure de nomadisme par une petite marche à pied. Nous comprendrions alors que le succès de Manheim est son invisibilité ; qu'à l'instar par exemple du «two-step flow» dont il aurait pu réclamer la paternité, il lui importait plus que sa découverte fut utile et soit utilisée que de figurer en bonne place dans les manuels de sociologie.

On se demandera peut-être en quoi un sociologue du «mainstream » américain, qui a tout fait pour effacer ses traces et pour ne pas revendiquer son " auctorialité » peut encore intéresser aujourd'hui. Je ne prendrai que l'exemple de la sociologie "publiciste » de Manheim. L'une de ses divergences avec Karl, outre le fait qu'il rejetait le magistère de l'intelligence libre de toute attache, résidait dans la «discursivité sociale » de l'opinion publique, dans le fait donc que cette opinion dépendait de relais collectifs - des discussions autour du zinc aux partis politiques - pour devenir effectif, et que l'influence politique était d'autant plus efficace que le mot d'ordre prenait en compte et anticipait les attentes de ces relais. Il est jusqu'à la récente campagne électorale française pour s'apercevoir de la justesse de cette analyse. Ainsi, la victoire de la droite n'est pas seulement la victoire d'un programme et d'un management politiques, mais la victoire d'une stratégie d'opinion qui a pris en compte ces relais intermédiaires. Elle a fourni au zinc, aux réunions de famille, aux débats entre collègues et amis des images (pensons à l'image de Ségolène Royal en madone), des slogans, des arguments, des mots d'ordre; alors que la stratégie de la candidate socialiste, portée par l'idée séduisante d'une campagne sur Internet, d'une rencontre intense avec les citoyens, n'avait pas bénéficié du martelage publicitaire mis en scène depuis des années par la candidate de la droite, ni pris en compte la nécessité d'articuler la parole politique sur une discursivité sociale. Que l'actionnisme politique se réduise finalement à une pratique de segmentation du marché des électeurs - voilà la leçon de Manheim - est évidemment une Realpolitik désidéalisée, mais ignorer parfois que tous les moyens du marketing sont bons pour parvenir à ses fins relève pour sa part de la faute professionnelle qu'aucune posture, aussi idéaliste fût-elle, ne saurait racheter. Ce à quoi Karl rétorquerait à juste titre : si aujourd'hui la «trahison des clercs » n'est plus possible - les seuls à s'exprimer durant cette campagne, finalement, furent les pitres sénescents de la «nouvelle philosophie »-, c'est que de clercs il n'y en a plus et que l'« intellect libre de toute attache » s'est évaporé dans les limbes.

\section{NOTES}

1. Après la mort de Leopold von Wiese, c'est l'élève de Freyer et d'Arnold Gehlen, Helmuth Schelsky, qui va se partager la fonction - avec René König - du «nestor» de la sociologie allemande. Le rôle institutionnel de Schelsky - notamment dans la création de l'Université de Bielefeld - sera considérable. Le personnage, cependant, est à plusieurs facettes. Car c'est de lui que provient la thèse que, si la sociologie allemande s'était si facilement laissée nazifier, c'est qu'elle avait tout bonnement cessé d'exister, qu'elle s'était épuisée (tout comme la République de Weimar...). Mais si elle avait été une proie facile, en somme, qu'y avait-il besoin alors de reprendre une sociologie sénile? De même, qu'y avait-il alors besoin d'incriminer les sociologues qui, comme Freyer, s'étaient « arrangés » avec les nazis? N'avaient-ils pas sauvé ce qu'il restait à 
sauver des décombres de la sociologie de Weimar ? Et c'est de lui que ressurgit le thème de l'antisociologie lors des événements de Mai 68 ; qu'en dehors d'une technologie sociale, toute forme de sociologie critique était tout bonnement anti-scientifique. Alors que l'attitude de Schelsky était jugée comme particulièrement réactionnaire, notamment suite aux interventions de René König - on lira avec profit ses interventions toniques dans Soziologie in Deutschland. Begründer - Verächter - Verfechter, Munich, Hanser, 1987, où le rôle du "Verächter ", du méprisant échoit à Schelsky précisément -, on assiste aujourd'hui à un retour en grâce d'un «néo-conservateur ", en mettant en avant ses travaux empiriques (sur la consommation, la famille, la sexualité) pour d'autant mieux camoufler son oeuvre de démolition idéologique de la sociologie.

2. Sa nièce, Elisabeth Werzig, nous donne une biographie passionnante dans Die Bewältigung der Mitte; Ernest Manheim : Soziologe und Anthropologe, Vienne, Böhlau, 1997. De même, sous un angle plus académique, on lira avec profit l'étude de Frank Baron, Authority, Culture and Communication. The Sociology of E. Manheim, Heidelberg, Wissenschaftsverlag der Autoren, 2005.

3. Ferdinand Tönnies, Der Selbstmord in Schleswig Holstein. Eine statistisch-soziologische Untersuchung, Breslau, Hirt, 1927.

4. Curieux personnage que ce Hans Freyer. Fondateur de l'École de Leipzig, d'où seront issus les meilleurs cadres de la sociologie nazie et une grande partie des sociologues conservateurs allemands d'après-Guerre (notamment Helmuth Schelsky), ce sociologue a une solide formation de philosophe, dont l'ouvrage fondateur, Theorie des objektiven Geistes (1928) qui poursuit les pensées de Hegel et de Wilhelm Dilthey, va préparer le projet sociologique, notamment dans son ouvrage Soziologie als Wirklichkeitswissenschaft (1930), d'une « révolution de droite » qui prendrait acte de l'anomie de la société industrielle et de l'échec de la lutte des classes, en lui opposant un Etat autoritaire. Ayant pris ses distances avec le nazisme - il sera professeur à Budapest entre 1941 et 1945 - il est l'exemple même du penseur conservateur, du théoricien de cette révolution conservatrice qui aura grand mal à se justifier au moment de la dénazification. Il n'en est pas moins l'un des premiers sociologues professionnels qui, après la mort de Max Weber et de Georg Simmel, dont il ne cesse de se nourrir de manière critique, va lancer des projets innovateurs en sociologie industrielle, des organisations et de l'administration publique. On lira avec profit les études d'Elfriede Uener sur l'École de Leipzig - non sans euphémisation sur l'implication de ses principaux représentants entre 1933 et 1945 : http://www.uener.com/kulturth.html.

5. Theodor Litt (1880-1962), l'un des principaux philosophes sociaux pendant la période de Weimar, et qui sera à l'origine de la pédagogie allemande moderne (Reformpädagogik). Son œuvre qui reste très largement à être redécouverte - on notera toutefois la publication en langue française de L'Individu et la communauté. Fondement philosophique de la culture, Lausanne, L’Âge d'homme, 1991 - est le modèle même de la dialectique individu/société, non sans référence constante au dialogisme d'un Martin Buber.

6. Exposé manuscrit de sa thèse «Die Logik des konkreten Begriffs ». L'original est déposé aux Archiv für die Geschichte der Soziologie in Österreich, à Graz, Nachlass Ernest Manheim, sign. 31/5. Première publication in Jahrbuch für Soziologiegeschichte, 1995, édité Carsten Klingemann, Michael Neumann, Karl-Siegbert Rehberg, Ilja Srubar, Erhard Stölting, éds., Opladen, Leske + Budrich, 1999, S. 25-26.

7. Die Träger der öffentlichen Meinung. Studien zur Soziologie der Öffentlichkeit. Brünn - Prague Leipzig - Vienne, Verlag Rudolf M. Rohrer, 1933, réédité sous le titre : Aufklärung und öffentliche Meinung. Studien zur Soziologie der Öffentlichkeit im 18. Jahrhundert. Dirigé et préfacé par Norbert Schindler. Stuttgart/Bad Cannstatt : frommann/holzboog 1979 (= Kultur und Gesellschaft, neue historische Forschungen. 4.).

8. On consultera là-dessus avec profit l'ouvrage de Volker Kruse, Historisch-soziologische Zeitdiagnosen in Westdeutschland nach 1945. Eduard Heimann, Alfred von Martin, Hans Freyer, Francfort, Suhrkamp, 1994. 
9. Une partie importante de la sociologie nazie va s'investir dans ce type d'empirie. Inutile de souligner que Manheim, vu son passé, n'a rien à voir avec ce que deviendra la sociologie allemande « auto-affiliée » au pouvoir.

10. On surestime généralement la carrière que fit Karl Mannheim dans son exil. Mis à part son rôle important dans le cercle Moot, Mannheim s'est très tôt heurté à Morris Ginsberg qui, à l'époque, était le mandarin de la sociologie britannique et qui l'a confiné dans un rôle subalterne à la LES. Ce n'est que trois mois avant sa mort que Mannheim retrouvera un poste de professeur ordinaire... en sciences de l'éducation à l'Université de Londres.

11. Archiv für die Geschichte der Soziologie in Österreich. Newsletter (Gravez), $\mathrm{n}^{\circ} 19$ (décembre 1999), pp. 20-27.

12. Risk, Uncertainty and Risk, Boston, MA, Schaffner, Marx \& Houghton, 1921.

13. Dans sa somme consacrée à l'École de Francfort, Rolf Wiggershaus n'évoque pas cet article, pas plus que Martin Jay ou Helmuth Dubiel. Il serait intéressant de savoir par quel biais Pollock a convoqué cet article et si Adorno en a fait usage dans ses Études sur la personnalité autoritaire, qui viennent d'être rééditées en France aux Éditions Allia, Paris, 2006.

14. Parmi les nombreux ouvrages qui fournissent des clés précieuses, j'en citerai simplement deux: celui de Sven Papcke, Deutsche Soziologie im Exil. Gegenwartsdiagnose und Epochenkritik (1933-1945), Francfort, Campus, 1933, qui nous livre des portraits éloquents de sociologues connus et complètement méconnus comme Leopold Schwarzschild, Fritz Sternberg, Adolf Löwe, Franz Neumann, Ernst Grünfeld, Peter Drucker (eh oui! le pape du management américain), Willy Strzelewicy, Karl Polányi et Albert Salomon; ainsi que l'ouvrage édité sous la direction d'Ilya Srbar, Exil, Wissenschaft, Identität; Die Emigration deutscher Sozialwissenschaftler (1933-1945), Francfort, Suhrkamp, 1988, avec notamment des contributions de Kurt H. Wolff et de Reinhard Bendix sur leur propre émigration, de Paul Neurath sur l'édification de la «multinationale Lazarsfeld ", de Claus-Dieter Krohn et de Benita Luckmann sur le rôle de la New School of Social Research, de Sigrid Ziffus sur l'implication de Karl Mannheim dans le cercle londonien "Moot » ou de Gunzelin Schmid Noerr sur l'émigration (plus ou moins dorée) de Max Horckheimer aux Etats-Unis.

15. Ainsi, Theodor W. Adorno nous montre dans ses «Wissenschaftliche Erfahrungen in Amerika ", in Gesammelte Schriften, t. 10/2, pp. 702-738, qu'une patrie loin de la patrie d'origine n'est pas nécessairement définitive, et que de ce double enracinement, dont l'un est toujours vécu comme une parenthèse de l'autre, peut naître un effet de diffraction qui rompt une fois pour toutes avec ce que je qualifierais d'ontologie de l'atterrement, avec le faux radicalisme heideggérien d'une métaphysique du lieu et de l'écoumène.

16. Un exemple parmi tant d'autres. En 1948, au bout de ses pérégrinations en Égypte et en Palestine, qui l'auront mené au bout du désespoir et de la désolation, le célèbre sociologue politique Siegfried Landshut qui avait découvert les Manuscrits philosophiques de Marx, croyait pouvoir enfin briguer un poste d'Ordinarius à l'Université de Hambourg, après qu'il en eut été chassé quinze années auparavant. Or voilà qu'il tombe sur la Doyen du Département de sociologie qui n'est que celui qui lui avait signifié - assez rudement - son congé en 1933. Et celui-ci de lui refuser son inscription au concours,... ne disposant pas d'informations assez précises sur son sujet. On trouvera tous les détails de ce scandale dans John Michael Krois, Gerhard Lohse et Rainer Nicolaysen, Die Wissenschaftler. Ernst Cassirer, Bruno Snell, Siegfried Landshut, Hambourg, Verlag Verein für Hamburgische Geschichte, 1994.

17. Karl Mannheim, « Testimonial for Ernest Manheim », décembre 1934, Archives de l'Université de Kansas City, Missouri, reproduit in : Stefanie Averbeck, «Ernst Manheim wissenschaftliches Jahrhundert : Erkenntnistheorie und Empirie », Archiv für die Geschichte der Soziologie in Oesterreich. Newsletter, 19, 1999, p. 3.

18. L'apport de Fritz K. Ringer (The Decline of German Mandarins. The German Academic Community, 1890-1933), Cambridge, Mass., Harvard University Press, 1969, pour cerner cette caste demeure 
exemplaire. Il permet, en effet, de préciser la coupure entre le mandarin Karl, encore préoccupé de jouer le rôle du "conseiller du prince ", et celui de son cousin qui n'est pas établi, ni dans la vie, ni dans sa conception de la société. Dans les termes de Ringer, Ernest fait déjà partie de ce monde post-mandarinal, ce monde où l'Ordinarius n'est plus l'autorité suprême, mais où il transmet simplement le savoir. À l'injonction charismatique du premier correspond la discursivité du second.

19. P. Lazarsfeld, B. Berelson, H. Gaudet (1944). The People's Choice. New York, Columbia University Press.

20. Ernest Manheim, "The role of small groups in the formation of public opinion ", South Western Social Science Quarterly, XX, 2, 1940, pp. 276-286, qui reprend une communication faite à Dallas en avril 1939.

\section{AUTEUR}

\section{ALDO HÄSLER}

Professeur de sociologie, directeur du département de sciences sociales de l'Université de Caen 